\title{
TITLE:
}

\section{Theoretical study of hydrogenated tetrahedral aluminum clusters}

\section{AUTHOR(S):}

Ichikawa, Kazuhide; Ikeda, Yuji; Wagatsuma, Ayumu; Watanabe, Kouhei; Szarek, Pawe; Tachibana, Akitomo

\section{CITATION:}

Ichikawa, Kazuhide ...[et al]. Theoretical study of hydrogenated tetrahedral aluminum clusters. International Journal of Quantum Chemistry 2010, 111(14): 3548-3555

\section{ISSUE DATE:}

2010-10-13

URL:

http://hdl.handle.net/2433/197380

\section{RIGHT:}

This is the peer reviewed version of the following article: Ichikawa, K., Ikeda, Y., Wagatsuma, A., Watanabe, K., Szarek, P. and Tachibana, A. (2011), Theoretical study of hydrogenated tetrahedral aluminum clusters. Int. J. Quantum Chem., 111:3548-3555, which has been published in final form at http://dx.doi.org/10.1002/qua.22848; This is not the published version. Please cite only the published version.; この論文は出版社版でありません。引用の際には出版社版 をご確認ご利用ください。 


\title{
Theoretical study of hydrogenated tetrahedral aluminum clusters
}

\author{
Kazuhide Ichikawa ${ }^{1}$, Yuji Ikeda ${ }^{1}$, Ayumu Wagatsuma ${ }^{1}$, \\ Kouhei Watanabe ${ }^{1}$, Paweł Szarek ${ }^{2}$ and Akitomo Tachibana*1 \\ ${ }^{1}$ Department of Micro Engineering, Kyoto University, Kyoto 606-8501, Japan \\ ${ }^{2}$ Wroctaw University of Technology, Institute of Physical and Theoretical Chemistry, \\ Wybrzeże Wyspiańskiego 27, 50-370 Wroctaw, Poland \\ E-mail: akitomo@scl.kyoto-u.ac.jp
}

\begin{abstract}
We report on the structures of aluminum hydrides derived from a tetrahedral aluminum $\left(\mathrm{Al}_{4}\right)$ cluster using ab initio quantum chemical calculation. Our calculation of binding energies of the aluminum hydrides reveals that stability of these hydrides increases as more hydrogen atoms are adsorbed, while stability of $\mathrm{Al}-\mathrm{H}$ bonds decreases. We also analyze and discuss the chemical bonds of those clusters by using recently developed method based on the electronic stress tensor.
\end{abstract}

wave function analysis; theory of chemical bond; stress tensor; hydrogenated aluminum cluster 


\section{Introduction}

Recently, hydrogen is paid much attention as new primary energy source because of the depletion of fossil fuels and environmental issues such as global warming. There are three basic research challenges - production, storage, application - for a "hydrogen economy". Our study in this paper is related to hydrogen storage. As is emphasized in the recent reports on basic research challenges for hydrogen storage, its high efficiency is a key factor in enabling the success of the hydrogen economy [1,2]. Hydrogen storage systems must exhibit following properties: appropriate thermodynamics, fast kinetics, high storage capacity (more than 10 wt \%), effective heat transfers, high volumetric densities, long cycle lifetime, safety under normal use [3]. To implement these properties, solid-state storage is useful. Metal hydrides, chemical storage materials, nanostructured materials are well known for effective solid-state storage systems.

Among them, we investigate aluminum hydrides in the form of aluminum clusters. More specifically, we deal with an aluminum tetrahedral cage $\left(\mathrm{Al}_{4}\right)$ and its hydrides which were recently observed in experiment and confirmed to have enhanced stability $[4,5]$. Although the clusters found in Refs. [4,5] are produced by vaporizing aluminum metal in hydrogen gas and do not lead to immediate application for hydrogen storage material at this stage, it would be useful to study their properties theoretically to further explore possible connection with more realistic materials for hydrogen storage. It is also interesting to study the tetrahedral cage in the aspect that it is the fundamental structure of an aluminum icosahedral cage $\left(\mathrm{Al}_{12}\right)$ [6]. In this paper, we report on the structures of aluminum hydrides which can be constructed by adding $\mathrm{H}_{2}$ molecules to an $\mathrm{Al}_{4}$ tetrahedral cluster.

We also evaluate and discuss the chemical properties of aluminum clusters and hydrogen adsorption by using a novel method of the electronic stress tensor based on the Regional Density Functional Theory (RDFT) and Rigged Quantum Electrodynamics (RQED) [719].

This paper is organized as follows. In the next section, we briefly explain our quantum chemical computation method. We also describe our analysis method based on the RDFT and the RQED, and in particular we define our bond orders and regional chemical potential. In Sec. 3, we discuss our results. Sec. 3.1 shows structures of hydrogenated $\mathrm{Al}_{4}$ tetrahedral clusters and discuss their binding energies. In Sec. 3.2, we analyze the structures using the electronic stress tensor and our bond orders. We summarize our paper in Sec. 4.

\section{Theory and calculation methods}

\subsection{Ab initio electronic structure calculation}

We perform ab initio quantum chemical calculation for several clusters of aluminum hydrides derived from an $\mathrm{Al}_{4}$ tetrahedral cage. In this work, calculations are performed by GAUSSIAN03 program package [20] using density functional theory (DFT) with Perdew- 
Wang 1991 exchange and correlation function (PW91) [21,22]. The split-valence triple-zeta 6-311G** basis set [23-25] with polarization functions has been used. Optimization was performed without imposing symmetry. The visualization is done using Visual Molecular Dynamics (VMD) [26] and PyMOL Molecular Viewer programs [27].

\section{$2.2 \quad$ RDFT analysis}

In the following section, we use quantities derived from the electronic stress tensor to analyze electronic structures of hydrogenated $\mathrm{Al}_{4}$ clusters. This method based on RDFT and RQED [7-13, 19] include useful quantities to investigate chemical bonding and reactivity such as new definition of bond order and regional chemical potential [14-16]. We briefly describe them below. (For other studies of quantum systems with the stress tensor in a slightly different context, see Refs. [28-39].)

The basic quantity in this analysis is the electronic stress tensor density $\overleftrightarrow{\tau} S(\vec{r})$ whose components are given by

$$
\begin{aligned}
\tau^{S k l}(\vec{r})= & \frac{\hbar^{2}}{4 m} \sum_{i} \nu_{i}\left[\psi_{i}^{*}(\vec{r}) \frac{\partial^{2} \psi_{i}(\vec{r})}{\partial x^{k} \partial x^{l}}-\frac{\partial \psi_{i}^{*}(\vec{r})}{\partial x^{k}} \frac{\partial \psi_{i}(\vec{r})}{\partial x^{l}}\right. \\
& \left.+\frac{\partial^{2} \psi_{i}^{*}(\vec{r})}{\partial x^{k} \partial x^{l}} \psi_{i}(\vec{r})-\frac{\partial \psi_{i}^{*}(\vec{r})}{\partial x^{l}} \frac{\partial \psi_{i}(\vec{r})}{\partial x^{k}}\right]
\end{aligned}
$$

where $\{k, l\}=\{1,2,3\}, m$ is the electron mass, $\psi_{i}(\vec{r})$ is the $i$ th natural orbital and $\nu_{i}$ is its occupation number.

Taking a trace of $\overleftrightarrow{\tau} S(\vec{r})$ can define energy density of the quantum system at each point in space. The energy density $\varepsilon_{\tau}^{S}(\vec{r})$ is given by

$$
\varepsilon_{\tau}^{S}(\vec{r})=\frac{1}{2} \sum_{k=1}^{3} \tau^{S k k}(\vec{r}) .
$$

We note that, by using the virial theorem, integration of $\varepsilon_{\tau}^{S}(\vec{r})$ over whole space gives usual total energy $E$ of the system: $\int \varepsilon_{\tau}^{S}(\vec{r}) d \vec{r}=E$.

Regional chemical potential $\mu_{R}[7]$ is calculated approximately using $\varepsilon_{\tau}^{S}(\vec{r})[14]$.

$$
\mu_{R}=\frac{\partial E_{R}}{\partial N_{R}} \approx \frac{\varepsilon_{\tau}^{S}(\vec{r})}{n(\vec{r})},
$$

where $n(\vec{r})$ is the ordinary electron density at $\vec{r}$. Since electrons tend to move from high $\mu_{R}$ region to low $\mu_{R}$ region, the distribution of $\mu_{R}$ maps the chemical reactivity.

Now, we define bond orders as $\varepsilon_{\tau}^{S}(\vec{r})$ or $\mu_{R}$ at "Lagrange point" [14]. The Lagrange point $\vec{r}_{L}$ is the point where the tension density $\vec{\tau}^{S}(\vec{r})$ given by the divergence of the stress 
tensor

$$
\begin{aligned}
\tau^{S k}(\vec{r})= & \sum_{l} \partial_{l} \tau^{S k l}(\vec{r}) \\
= & \frac{\hbar^{2}}{4 m} \sum_{i} \nu_{i}\left[\psi_{i}^{*}(\vec{r}) \frac{\partial \Delta \psi_{i}(\vec{r})}{\partial x^{k}}-\frac{\partial \psi_{i}^{*}(\vec{r})}{\partial x^{k}} \Delta \psi_{i}(\vec{r})\right. \\
& \left.+\frac{\partial \Delta \psi_{i}^{*}(\vec{r})}{\partial x^{k}} \psi_{i}(\vec{r})-\Delta \psi_{i}^{*}(\vec{r}) \frac{\partial \psi_{i}(\vec{r})}{\partial x^{k}}\right]
\end{aligned}
$$

vanishes. Namely, $\tau^{S k}\left(\vec{r}_{L}\right)=0 . \quad \vec{\tau}^{S}(\vec{r})$ is the expectation value of the tension density operator $\hat{\vec{\tau}}^{S}(\vec{r})$, which cancels the Lorentz force density operator $\hat{\vec{L}}(\vec{r})$ in the equation of motion for stationary state [11]. Therefore, we see that $\vec{\tau}^{S}(\vec{r})$ expresses purely quantum mechanical effect and it has been proposed that this stationary point might characterize chemical bonding [14]. Then, our newly defined bond orders are

$$
b_{\varepsilon}=\frac{\varepsilon_{\tau \mathrm{AB}}^{S}\left(\vec{r}_{L}\right)}{\varepsilon_{\tau \mathrm{HH}}^{S}\left(\vec{r}_{L}\right)},
$$

and

$$
b_{\mu}=\frac{\varepsilon_{\tau \mathrm{AB}}^{S}\left(\vec{r}_{L}\right) / n_{\mathrm{AB}}\left(\vec{r}_{L}\right)}{\varepsilon_{\tau \mathrm{HH}}^{S}\left(\vec{r}_{L}\right) / n_{\mathrm{HH}}\left(\vec{r}_{L}\right)} .
$$

One should note normalization by the respective values of a $\mathrm{H}_{2}$ molecule calculated at the same level of theory (including method and basis set).

We use Molecular Regional DFT (MRDFT) package [40] to compute these quantities introduced in this section.

\section{Results and discussion}

\subsection{Structures and stability}

The bare tetrahedral optimized structure of $\mathrm{Al}_{4}$ is shown in Fig. 1 (a). We found that all the six $\mathrm{Al}-\mathrm{Al}$ bonds have an equal length of $2.74 \AA$ to a great accuracy. $\mathrm{Al}_{4}$ is considered to have a structure very close to a regular tetrahedron. We note that this regular tetrahedral structure is stable only for high spin state (multiplicity $=5$ ). Also it should be noted that the global minimum of $\mathrm{Al}_{4}$ cluster is planar rhombus with multiplicity 3 [41, 42], which has lower energy by $0.5 \mathrm{eV}$. Below, we investigate the structures when hydrogens adsorbs to this tetrahedral structure.

We first considered the adsorption of two hydrogen atoms. We examined many combinations of adsorption sites and multiplicities 1,3 and 5 . We found that $\mathrm{Al}_{4} \mathrm{H}_{2}$ with two 
hydrogen atoms at terminal sites as shown in Fig. 1 (b) and with multiplicity 3, has the lowest energy.

We further added hydrogen atoms to this structure. Fig. 1 (c)-(g) show structures of most stable isomer of $\mathrm{Al}_{4} \mathrm{H}_{n}(n=4,6,8,10$ and 12) we have obtained. We have tried to adsorb more hydrogen, without success. We could not find a stable structure for $\mathrm{Al}_{4} \mathrm{H}_{14}$. Thus, we believe $\mathrm{Al}_{4} \mathrm{H}_{12}$ is the saturated structure of the $\mathrm{Al}_{4}$ cluster.

Here, we comment on the comparison with the structures which were reported in literatures. The structure of $\mathrm{Al}_{4} \mathrm{H}_{4}$, Fig. 1 (c), is consistent with Ref. [5,43] and $\mathrm{Al}_{4} \mathrm{H}_{6}$, Fig. $1(\mathrm{~d})$, is consistent with Ref. [5]. As for $\mathrm{Al}_{4} \mathrm{H}_{12}$, there are several literatures which investigated stable structures for this as a tetramer of alane $\mathrm{Al} \mathrm{H}_{3}$ [43-46]. This structure also attracts interest because of its high hydrogen storage capacity of $10.0 \mathrm{wt} \%$, which exceeds the target value of a hydrogen storage system specified by some technical report [3]. Ref. [43] has pointed out that structures in Refs. [44] and [45] are not stable in light of recent quantum chemical computation and reported a structure with $\mathrm{S}_{4}$ symmetry as the global minimum. Our results, Fig. 1 (g), agrees with the structure found in Ref. [43]. Ref. [43] also reported stable structures of $\mathrm{Al}_{4} \mathrm{H}_{10}$, including the structure we have found as Fig. 1 (f). They have shown that there is a structure with lower energy but since that structure is chain-like, we adopt the structure of Fig. 1 (f) as the one which is derived by adding hydrogen atoms to Fig. 1 (e).

In order to investigate the stability of these structures, we here define two types of binding energies (B.E.). The total B.E. of $\mathrm{Al}_{4} \mathrm{H}_{m}$ is

$$
\Delta E_{\text {total }}=E\left(\mathrm{Al}_{4} \mathrm{H}_{m}\right)-[4 E(\mathrm{Al})+m E(\mathrm{H})],
$$

where $E(\mathrm{X})$ is the energy of $\mathrm{X} . \Delta E_{\text {total }}$ represents the sum of the strength of all the bonds existing in the molecule. The average B.E. of $\mathrm{H}$ atoms is defined as

$$
\Delta E_{\mathrm{H}}=\frac{1}{m}\left\{E\left(\mathrm{Al}_{4} \mathrm{H}_{m}\right)-\left[E\left(\mathrm{Al}_{4}\right)+m E(\mathrm{H})\right]\right\}
$$

which represents the strength of $\mathrm{Al}-\mathrm{H}$ bond per one hydrogen atom. We use the structure of Fig. 1 (a) to calculate $E\left(\mathrm{Al}_{4}\right)$.

The results for these two types of binding energy are summarized in Table 1 and Fig. 2. As is shown in Fig. 2 (a), $\Delta E_{\text {total }}$ decreases as more hydrogens are adsorbed. This indicates that adsorption of hydrogen stabilizes the cluster. On the other hand, as we plot in Fig. 2 (b), $\Delta E_{\mathrm{H}}$ is almost same for $\mathrm{Al}_{4} \mathrm{H}_{2}$ and $\mathrm{Al}_{4} \mathrm{H}_{4}$ but increases as more hydrogens are adsorbed. This is considered to be due to the increased hydrogens at bridge sites for larger clusters than $\mathrm{Al}_{4} \mathrm{H}_{6}$. It should be stressed that lower total B.E. and higher average B.E. of $\mathrm{H}$ atoms are favorable for hydrogen storage systems. This means that hydrogen atoms turn into state such that they are easily desorbed, while clusters become more stable as hydrogen are adsorbed. 


\subsection{Stress tensor analysis of chemical bond}

In the previous subsection, we have shown structures of aluminum hydrides derived from the $\mathrm{Al}_{4}$ tetrahedral cage and discussed the stability of these clusters by the usual binding energy. In this section, we discuss their chemical bonds using RDFT analysis introduced in Sec. 2.2.

Actually, we have already used the RDFT concept to draw Fig. 1. There, we draw bond lines when the Lagrange point is found between two atoms. The Lagrange point is the point at which tension density vanishes (Sec. 2.2) and considered to be suitable to define chemical bond [14-16]. Two types of bond orders, $b_{\varepsilon}$ (Eq. (5)) and $b_{\mu}$ (Eq. (6)), are computed and summarized as functions of bond distance in Fig. 3. The $b_{\varepsilon}$ describes bond strength in relation to bond in $\mathrm{H}_{2}$ molecule and how particular bond contributes to lowering of total energy of a structure, whereas $b_{\mu}$ shows bond electrophilicity in relation to $\mathrm{H}_{2}$ molecule as reference bond.

In the figure, we distinguish different types of bonds. $\mathrm{Al}-\mathrm{Al}$ bonds with and without bridging hydrogen are denoted by "Al-Al(b)" and "Al-Al". Al-H bonds at terminal sites are denoted as "Al- $\mathrm{H}(\mathrm{t})$ " and those at bridge sites are denoted as "Al-H(b)". We find good correlation between bond distance and our bond orders as has been found in other molecules [14-16]. $b_{\varepsilon}$ and $b_{\mu}$ basically exhibit similar features regarding the correlation between bond length in the sense that the slope for $\mathrm{Al}-\mathrm{Al}$ bonds are larger than $\mathrm{Al}-\mathrm{H}$ bonds. There is a subtle difference in $\mathrm{Al}-\mathrm{H}$ bonds between $b_{\varepsilon}$ and $b_{\mu}$. For $b_{\varepsilon}, \mathrm{Al}-\mathrm{H}(\mathrm{t})$ and Al-H(b) together make a single slope but there seems to appear three families of slopes for $b_{\mu}$. Since $b_{\mu}$ concerns the regional chemical potential, the difference may reflect the chemical reactivity of these bonds.

We can further examine features in chemical bonds by analyzing the electronic stress tensor (Eq. (1)). For example, hydrogen bridged $\mathrm{Al}-\mathrm{Al}$ bonds are investigated for $\mathrm{Al}_{4} \mathrm{H}_{8}$, $\mathrm{Al}_{4} \mathrm{H}_{10}$ and $\mathrm{Al}_{4} \mathrm{H}_{12}$. Figs. 4, 5 and 6 plot the largest eigenvalue of the stress tensor and corresponding eigenvector on a plane which includes three atoms which participate in the bridging bonds, respectively for $\mathrm{Al}_{4} \mathrm{H}_{8}, \mathrm{Al}_{4} \mathrm{H}_{10}$ and $\mathrm{Al}_{4} \mathrm{H}_{12}$. Since the eigenvectors have three spatial components, namely they are 3D objects, we express them by projecting on the plane. In all these clusters, there are Lagrange points between $\mathrm{Al}$ and $\mathrm{H}$. There is a region with positive eigenvalue of the stress tensor (tensile stress) between them, which is typical for covalent bond involving $\mathrm{H}$ atom $[12,18]$. Also, there is a flow of corresponding eigenvectors connecting $\mathrm{Al}$ and $\mathrm{H}$ which indicates a formation of strong bonding.

For $\mathrm{Al}_{4} \mathrm{H}_{8}$, which has a Lagrange point between $\mathrm{Al}$ atoms, also has a flow of eigenvectors between them. In contrast to the case of Al-H bond, the eigenvalue of the stress tensor turns out to be negative (compressive stress) indicating a different nature of bonding. In the case of $\mathrm{Al}_{4} \mathrm{H}_{10}$, we did not find a Lagrange point between $\mathrm{Al}$ atoms. In Fig. 5, there is a similar flow of eigenvectors as in Fig. 4 between $\mathrm{Al}$ atoms, but this structure is shifted off the region between $\mathrm{Al}$ atoms likely to be because the $\mathrm{H}$ atom came close to $\mathrm{Al}$ atoms. Therefore we may conclude there is not so much direct interaction between $\mathrm{Al}$ atoms as to call bonding. We can reach similar conclusion for the case of $\mathrm{Al}_{4} \mathrm{H}_{12}$. Fig. 6 shows similar 
structure but it is further away from the region between $\mathrm{Al}$ atoms and in fact (when we see the flow in 3D) the flow is connected to $\mathrm{H}(11)$ atom which locates at slightly off this plane. This indicates that $\mathrm{Al}-\mathrm{Al}$ bonding is completely disrupted by the existence of $\mathrm{H}$ atoms.

Another example of the electronic stress tensor analysis is provided for $\mathrm{Al}_{4} \mathrm{H}_{10}$ in Fig. 7 . This concerns the somewhat radical structural change between $n=0,2,4,6,8$ and $n=$ 10, 12 of $\mathrm{Al}_{4} \mathrm{H}_{n}$. As shown in Fig. 1, in terms of Lagrange points, four Al atoms form a tetrahedral cage for $n \leq 8$ and but the cage seems to be broken for $n \geq 10$. This is also seen in a jump in the average Al-Al distance (Table 1). We can confirm this by analyzing the stress tensor density. As is shown in Fig. 7, there is a region between $\mathrm{Al}(2)$ and $\mathrm{Al}(3)$ in which eigenvectors go perpendicular to the plane (so that expressed by dots), showing total disconnection of these atoms.

\section{Summary}

In this paper, we investigated the structures of aluminum hydrides derived from a tetrahedral aluminum $\left(\mathrm{Al}_{4}\right)$ cluster using ab initio quantum chemical calculation. We reported stable structures of $\mathrm{Al}_{4} \mathrm{H}_{n}(n=0,2,4,6,8,10$ and 12), which include structures already found in the literature who had investigated the hydrogenated aluminum clusters from other aspects. We calculated binding energies of the aluminum hydrides and found interesting properties as hydrogen storage material: stability of the clusters increases as more hydrogen atoms are adsorbed, while stability of $\mathrm{Al}-\mathrm{H}$ bonds decreases.

We also analyzed and discussed the chemical bonds of those clusters by using the electronic stress tensor. The bond orders defined from energy density and regional chemical potential (which are in turn calculated from the stress tensor) are shown to have a good correlation with respect to the bond distance and to be able to distinguish types of bonding to some extent. As far as metallic elements are concerned, our bond order analysis had been only applied to Pt clusters [16] so the present analysis can be useful basis for further research using our stress tensor based analysis. This is also true for the eigenvalue and eigenvector analysis of the stress tensor. We have found that $\mathrm{Al}-\mathrm{H}$ bonds have a positive eigenvalue (tensile stress) at the region between the atoms where as $\mathrm{Al}-\mathrm{Al}$ bonds have a negative value. This indicates that the stress tensor can be a powerful tool to classify chemical bonding and may provide a deeper insight into the nature of chemical bonds.

\section{References}

[1] C. Read, J. Petrovic, G. Ordaz, and S. Satyapal, Mater. Res. Soc. Symp. Proc. 885A, warrendale, PA (2006).

[2] Report by ACIL Tasman and Parsons Brinckerhoff for Australian Government: "National Hydrogen Study," (2003). 
[3] Argonne National Laboratory. Report on Basic Energy Sciences Workshop on Hydrogen Production, Storage, and Use "Basic Research Needs for the Hydrogen Economy," May 13-15, 2003.

[4] X. Li et al., Science 315, 356 (2007).

[5] A. Grubisic, J. Am. Chem. Soc., 129 (18), 5969 (2007)

[6] A. Goldberg and I. Yarovsky, Phys. Rev. B 75, 195403 (2007).

[7] A. Tachibana, Theor. Chem. Acc. 102, 188 (1999).

[8] A. Tachibana, J. Chem. Phys. 115, 3497 (2001).

[9] A. Tachibana, in Stress Induced Phenomena in Metallization, ed. by S. P. Baker, (American Institute of Physics, New York, 2002), p. 105.

[10] A. Tachibana, in Reviews in Modern Quantum Chemistry, A Celebration in the Contributions of Robert Parr, ed. by K. D. Sen, (World Scientific, Singapore, 2002), Chap. 45, p. 1327.

[11] A. Tachibana, in Fundamental World of Quantum Chemistry, A Tribute to the Memory of Per-Olov Löwdin, ed. by E. J. Brändas and E. S. Kryachko, (Kluwer Academic, Dordrecht, 2003), Vol. 2, p. 211.

[12] A. Tachibana, Int. J. Quant. Chem. 100, 981 (2004).

[13] A. Tachibnana, J. Mol. Model. 11301 (2005).

[14] P. Szarek and A. Tachibana, J. Mol. Model,13, 651 (2007).

[15] P. Szarek, Y. Sueda, and A. Tachibana, J. Chem. Phys. 129, 094102 (2008).

[16] P. Szarek, K. Urakami, C. Zhou, H. Cheng, and A. Tachibana, J. Chem. Phys. 130, 084111 (2009).

[17] K. Ichikawa, T. Myoraku, A. Fukushima, Y. Ishihara, R. Isaki, T. Takeguchi and A. Tachibana, J. Mol. Struct. (THEOCHEM) 915, 1 (2009).

[18] K. Ichikawa and A. Tachibana, Phys. Rev. A 80, 062507 (2009).

[19] A. Tachibana, J. Mol. Struct. (THEOCHEM), 943, 138 (2010).

[20] M. J. Frisch et al., Gaussian 03, Revision B.05, Gaussian, Inc., Pittsburgh PA (2003).

[21] D. R. Hamann, Phys. Rev. B 40, 2980 (1989).

[22] J. P. Perdew and Y. Wang, Phys. Rev. B 45, 13244 (1992). 
[23] R. C. Binning Jr. and L. A. Curtiss, J. Comp. Chem. 11, 1206 (1990).

[24] M. P. McGrath and L. Radom, J. Chem. Phys. 94, 511 (1991).

[25] L. A. Curtiss, M. P. McGrath, J.-P. Blaudeau, N. E. Davis, R. C. Binning Jr., and L. Radom, J. Chem. Phys. 103, 6104 (1995).

[26] W. Humphrey, A. Dalke, and K. Schulten, J. Molec. Graphics, 14, 33 (1996).

[27] W. L. DeLano, The PyMOL Molecular Graphics System. (2008) DeLano Scientific LLC, Palo Alto, CA, USA. http://www.pymol.org

[28] R. F. W. Bader, J. Chem. Phys. 73, 2871 (1980).

[29] O. H. Nielsen and R. M. Martin, Phys. Rev. Lett. 50, 697 (1983).

[30] O. H. Nielsen and R. M. Martin, Phys. Rev. B 32, 3780 (1985).

[31] N. O. Folland, Phys. Rev. B 34, 8296 (1986).

[32] N. O. Folland, Phys. Rev. B 34, 8305 (1986).

[33] M. J. Godfrey, Phys. Rev. B 37, 10176 (1988).

[34] A. Filippetti and V. Fiorentini, Phys. Rev. B 61, 8433 (2000).

[35] A. M. Pendás, J. Chem. Phys. 117, 965 (2002).

[36] C. L. Rogers and A. M. Rappe, Phys. Rev. B 65224117 (2002).

[37] S. Morante, G. C. Rossi, and M. Testa, J. Chem. Phys. 125, 034101 (2006).

[38] J. Tao, G. Vignale, and I. V. Tokatly, Phys. Rev. Lett. 100, 206405 (2008).

[39] P. W. Ayers and S. Jenkins, J. Chem. Phys. 130, 154104 (2009).

[40] M. Senami, K. Ichikawa, K. Doi, P. Szarek, K. Nakamura, and A. Tachibana, Molecular Regional DFT program package, ver. 3. Tachibana Lab, Kyoto University, Kyoto (2008).

[41] R. O. Jones, Phys. Rev. Lett. 67, 224 (1991).

[42] H. Kawamura, V. Kumar, Q. Sun and Y. Kawazoe, Phys. Rev. B65 045406 (2001).

[43] J. Moc, K. Bober, and K. Mierzwicki, Chem. Phys. 327, 247 (2006).

[44] M. Shen, C. Liang, and H. F. Schaefer III, Chem. Phys. 171325 (1993).

[45] B. G. Willis and K. F. Jensen, J. Phys. Chem. A 1047881 (2000).

[46] H. Kawamura, V. Kumar, Q. Sun and Y. Kawazoe, Phys. Rev. A67 063205 (2003). 
Table 1: Total B.E. $\Delta E_{\text {total }}$, average B.E. of $\mathrm{H}$ atoms $\Delta E_{\mathrm{H}}$ and mean nearest-neighbor bond lengths $d_{\mathrm{x}-\mathrm{x}}$ of $\mathrm{Al}_{4} \mathrm{H}_{n}(n=2,4,6,8,10$ and 12$) . \mathrm{H}(\mathrm{t})$ and $\mathrm{H}(\mathrm{b})$ denote a hydrogen at the terminal site and the bridge site respectively.

\begin{tabular}{lccccc}
\hline \hline & $\Delta E_{\text {total }}(\mathrm{eV})$ & $\Delta E_{\mathrm{H}}(\mathrm{eV})$ & $d_{\mathrm{Al}-\mathrm{Al}}(\AA)$ & $d_{\mathrm{Al}-\mathrm{H}(\mathrm{t})}(\AA)$ & $d_{\mathrm{Al}-\mathrm{H}(\mathrm{b})}(\AA)$ \\
\hline $\mathrm{Al}_{4}$ & -5.59 & - & 2.74 & - & - \\
$\mathrm{Al}_{4} \mathrm{H}_{2}$ & -11.5 & -2.94 & 2.66 & 1.61 & - \\
$\mathrm{Al}_{4} \mathrm{H}_{4}$ & -17.3 & -2.94 & 2.60 & 1.61 & - \\
$\mathrm{Al}_{4} \mathrm{H}_{6}$ & -22.7 & -2.84 & 2.63 & 1.60 & 1.74 \\
$\mathrm{Al}_{4} \mathrm{H}_{8}$ & -27.6 & -2.75 & 2.69 & 1.59 & 1.77 \\
$\mathrm{Al}_{4} \mathrm{H}_{10}$ & -32.2 & -2.66 & 3.11 & 1.59 & 1.74 \\
$\mathrm{Al}_{4} \mathrm{H}_{12}$ & -36.6 & -2.59 & 3.47 & 1.59 & 1.72 \\
\hline
\end{tabular}


(a)

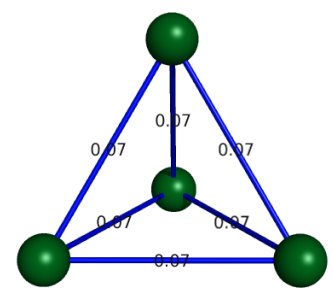

(b)

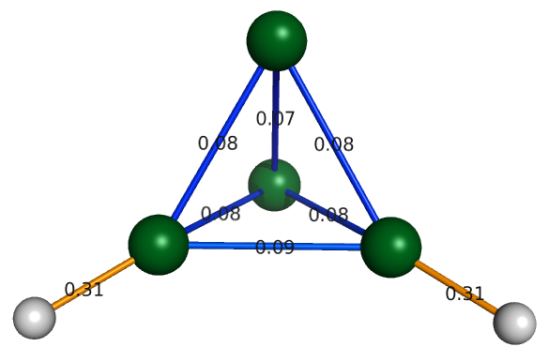

(d)

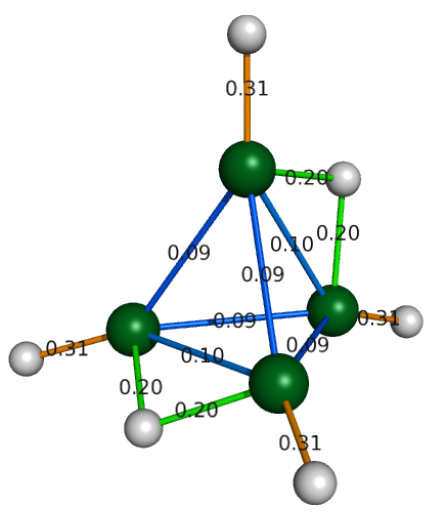

(f)

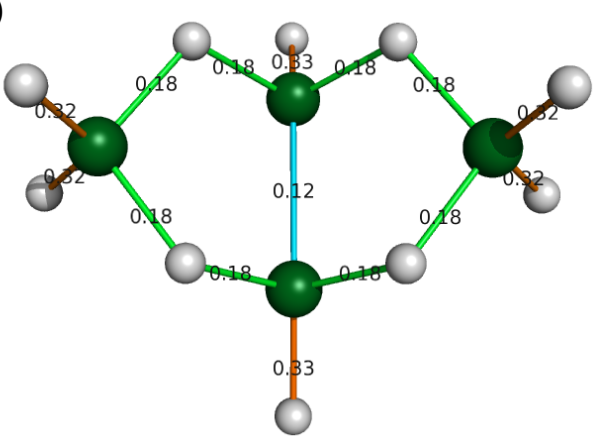

(c)
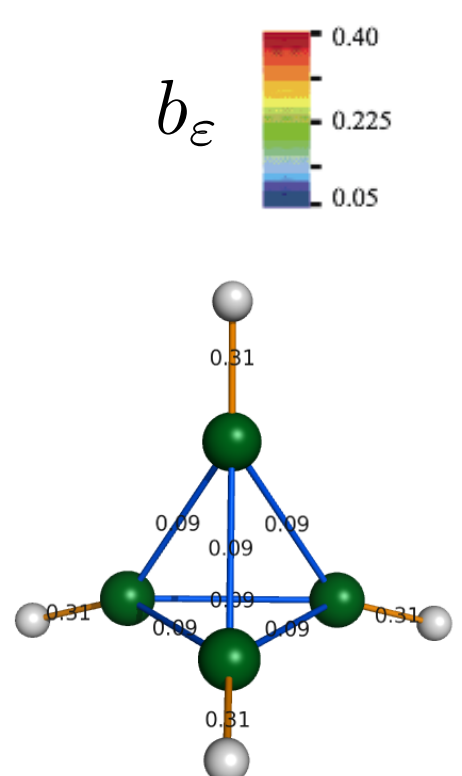

(e)

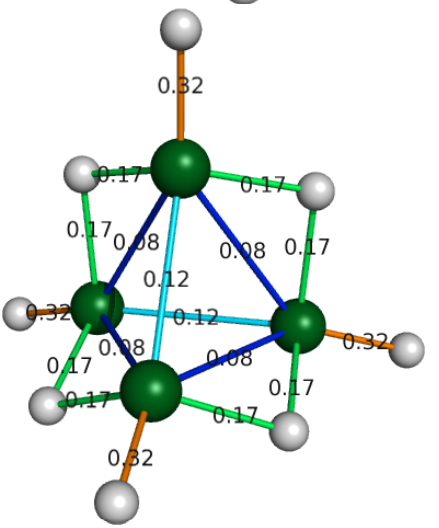

(g)

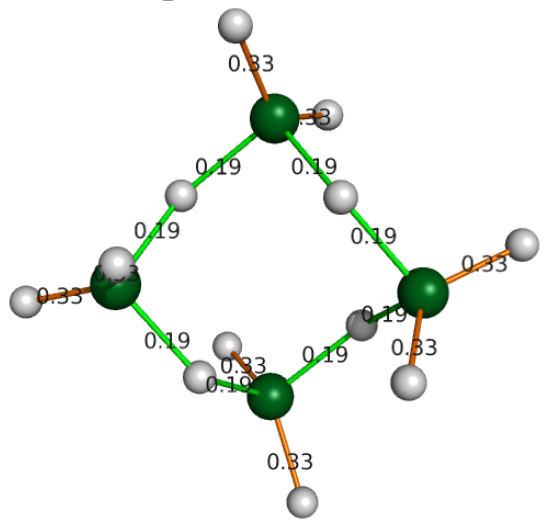

Figure 1: Optimized structures for $\mathrm{Al}_{4}$ tetrahedral cage and its hydrides $\mathrm{Al}_{4} \mathrm{H}_{n}$ ( $n=$ $2,4,6,8,10,12)$. The bonds are drawn at which Lagrange points are found and our energy density based bond order $b_{\varepsilon}$ is shown. 

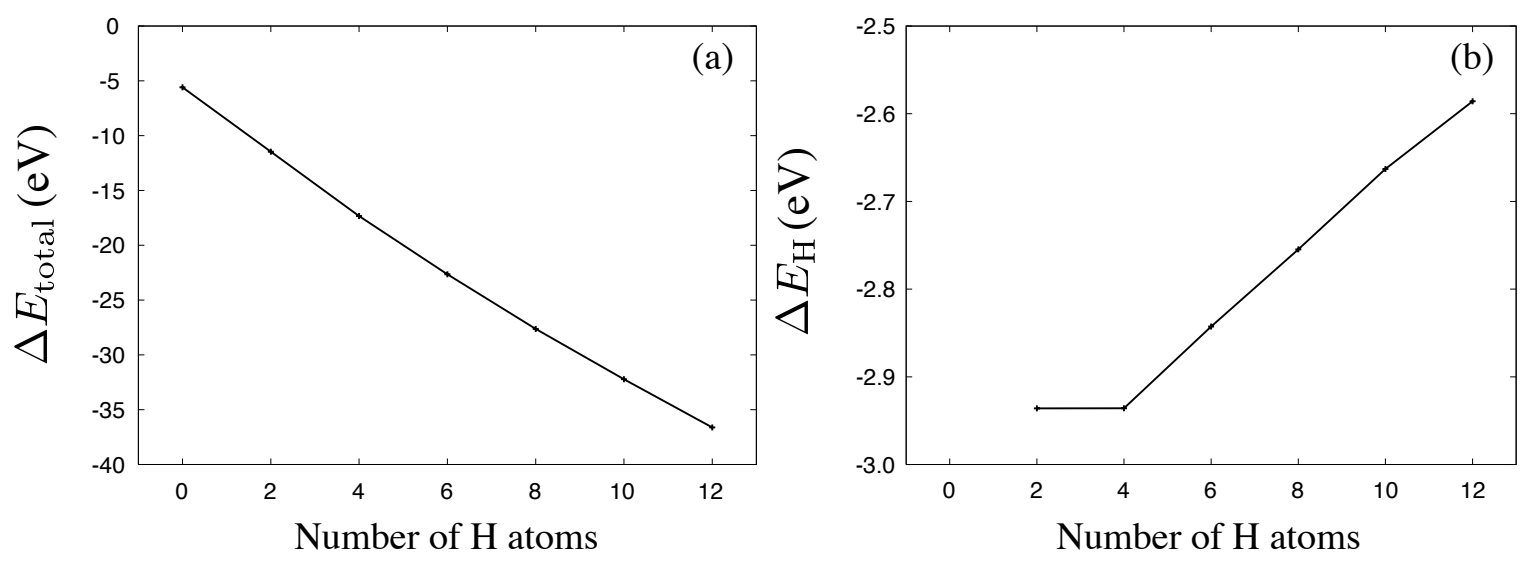

Figure 2: Total B.E. $\Delta E_{\text {total }}(\operatorname{panel}(\mathrm{a}))$ and average B.E. of $\mathrm{H}$ atoms $\Delta E_{\mathrm{H}}($ panel $(\mathrm{b}))$ of $\mathrm{Al}_{4} \mathrm{H}_{n}(n=0,2,4,6,8,10$ and 12).
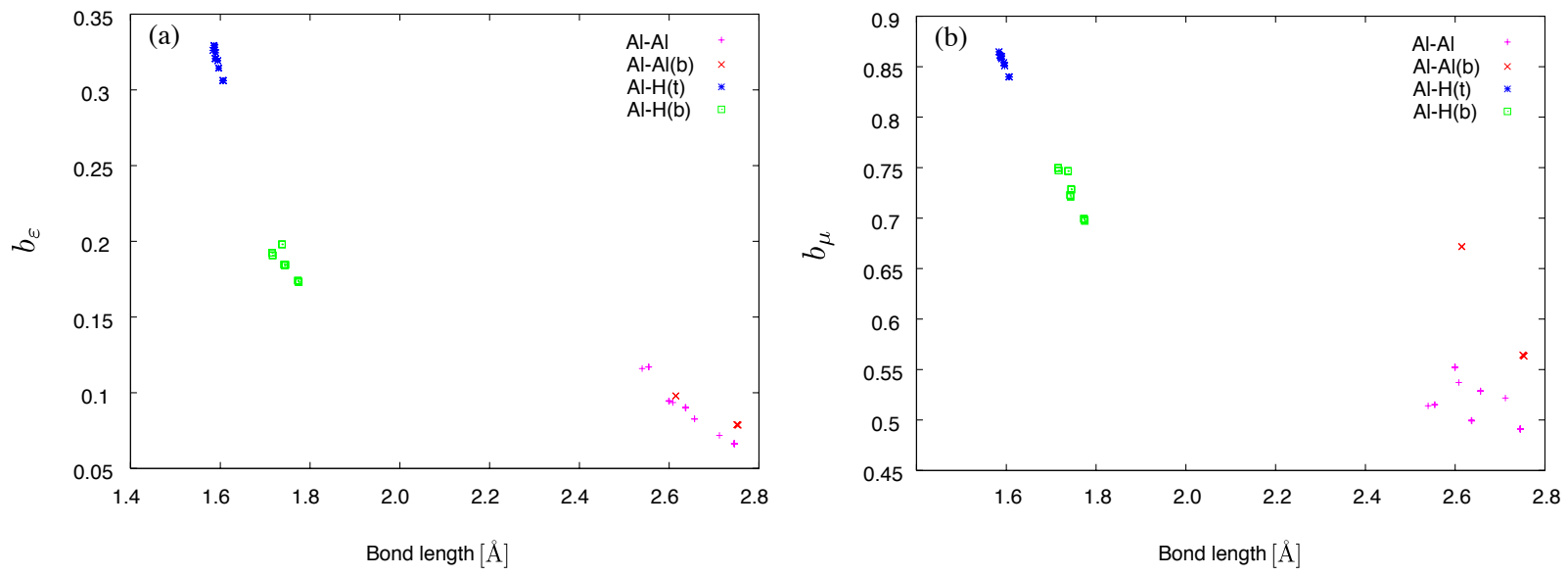

Figure 3: Energy density bond order $b_{\varepsilon}$ (panel (a)) and chemical potential bond order $b_{\mu}$ (panel (b)) as functions of bond length. Data are taken from the structures of $\mathrm{Al}_{4} \mathrm{H}_{n}$ ( $n=0,2,4,6,8,10$ and 12$)$ as shown in Fig. 1 . Al-Al bonds with bridging hydrogen are plotted with red crosses and those without are plotted with magenta plus marks. Al- $\mathrm{H}$ bonds at terminal sites are plotted with blue asterisks and those at bridge sites are plotted with green squares. 

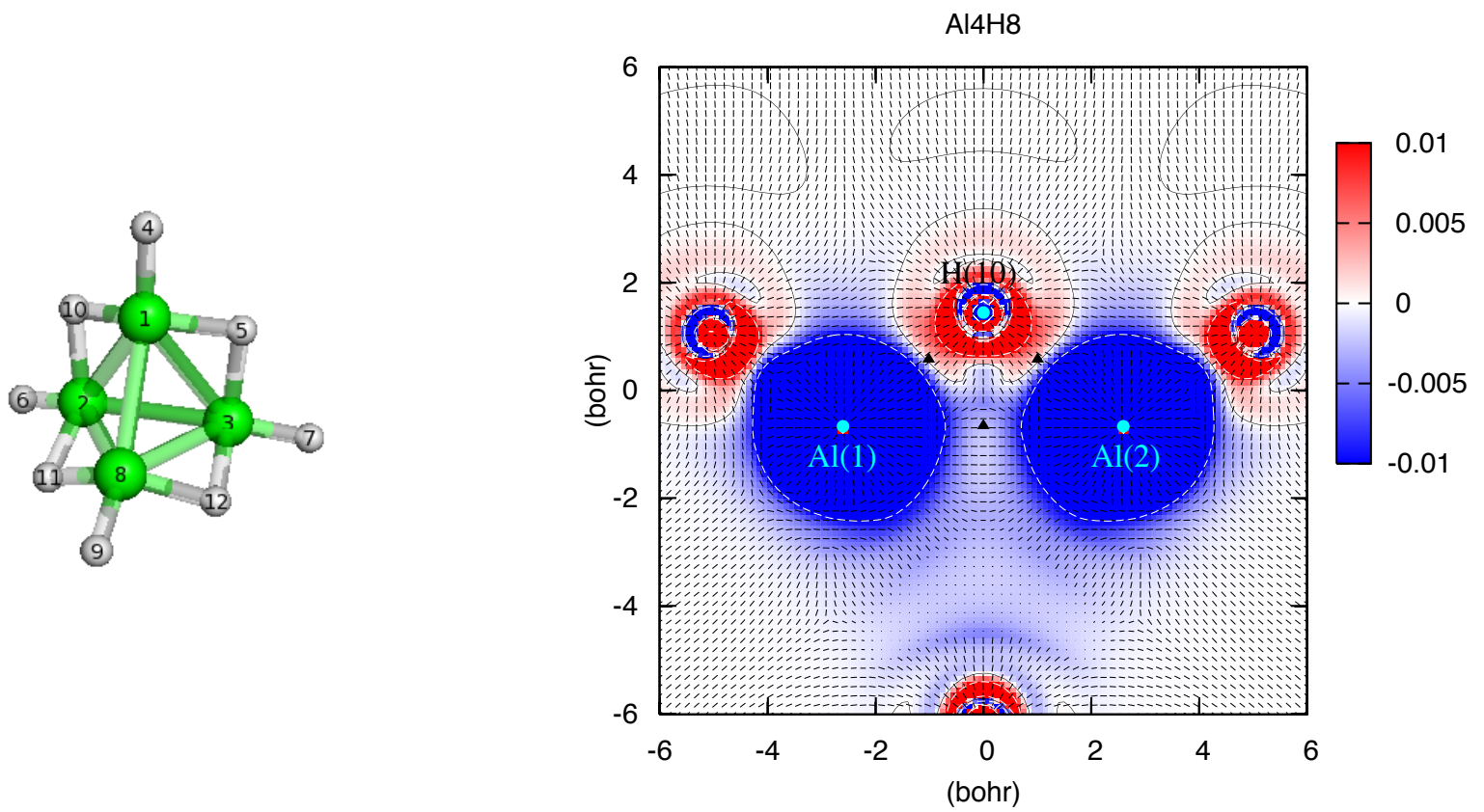

Figure 4: The largest eigenvalue of the stress tensor and corresponding eigenvector of $\mathrm{Al}_{4} \mathrm{H}_{8}$ on the right panel. They are shown on a plane which includes three labeled atoms. As for the eigenvectors, the projection on this plane is plotted. The positions of these atoms are shown by the circle dots. Parenthesized numbers in the labels correspond to the numbers on atoms on the left panel. As for the eigenvalue, we only show for the range $[-0.01,0.01]$ with color scale shown on the right and the contours for 0.01 and -0.01 are shown by white dashed lines. The triangle dots shows the locations of the Lagrange points. 

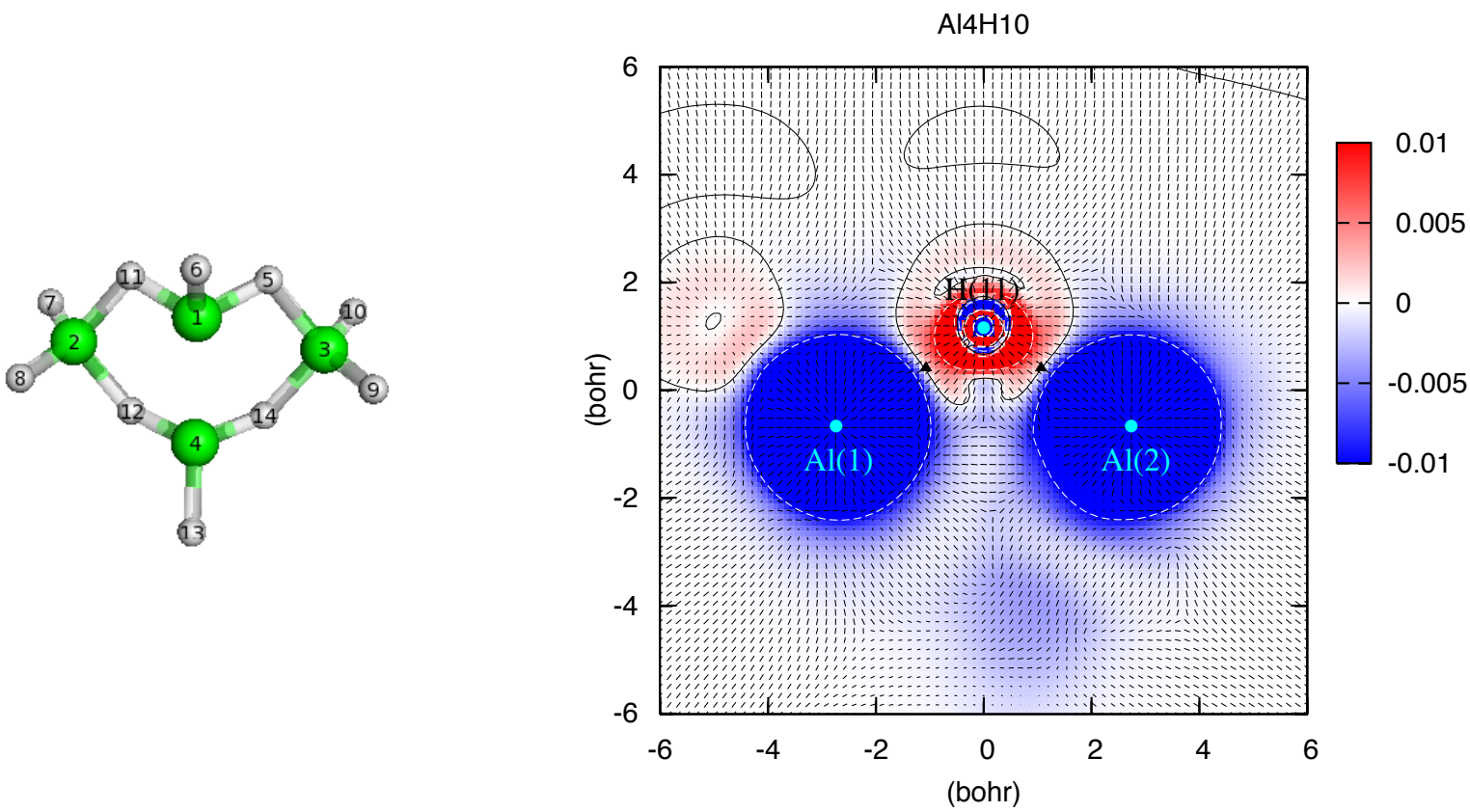

Figure 5: The largest eigenvalue of the stress tensor and corresponding eigenvector of $\mathrm{Al}_{4} \mathrm{H}_{10}$, plotted in the same manner as Fig. 4 .
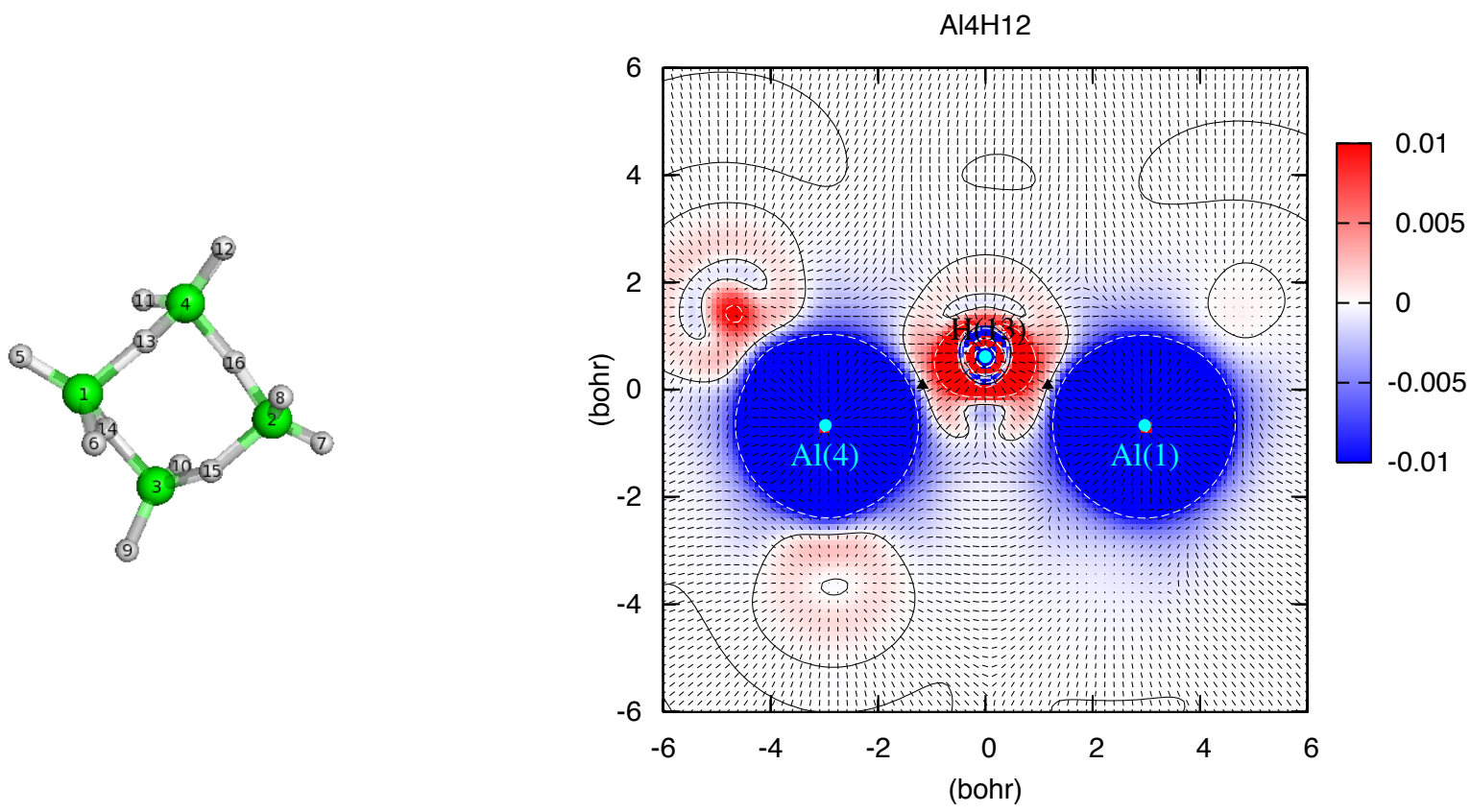

Figure 6: The largest eigenvalue of the stress tensor and corresponding eigenvector of $\mathrm{Al}_{4} \mathrm{H}_{12}$, plotted in the same manner as Fig. 4 . 

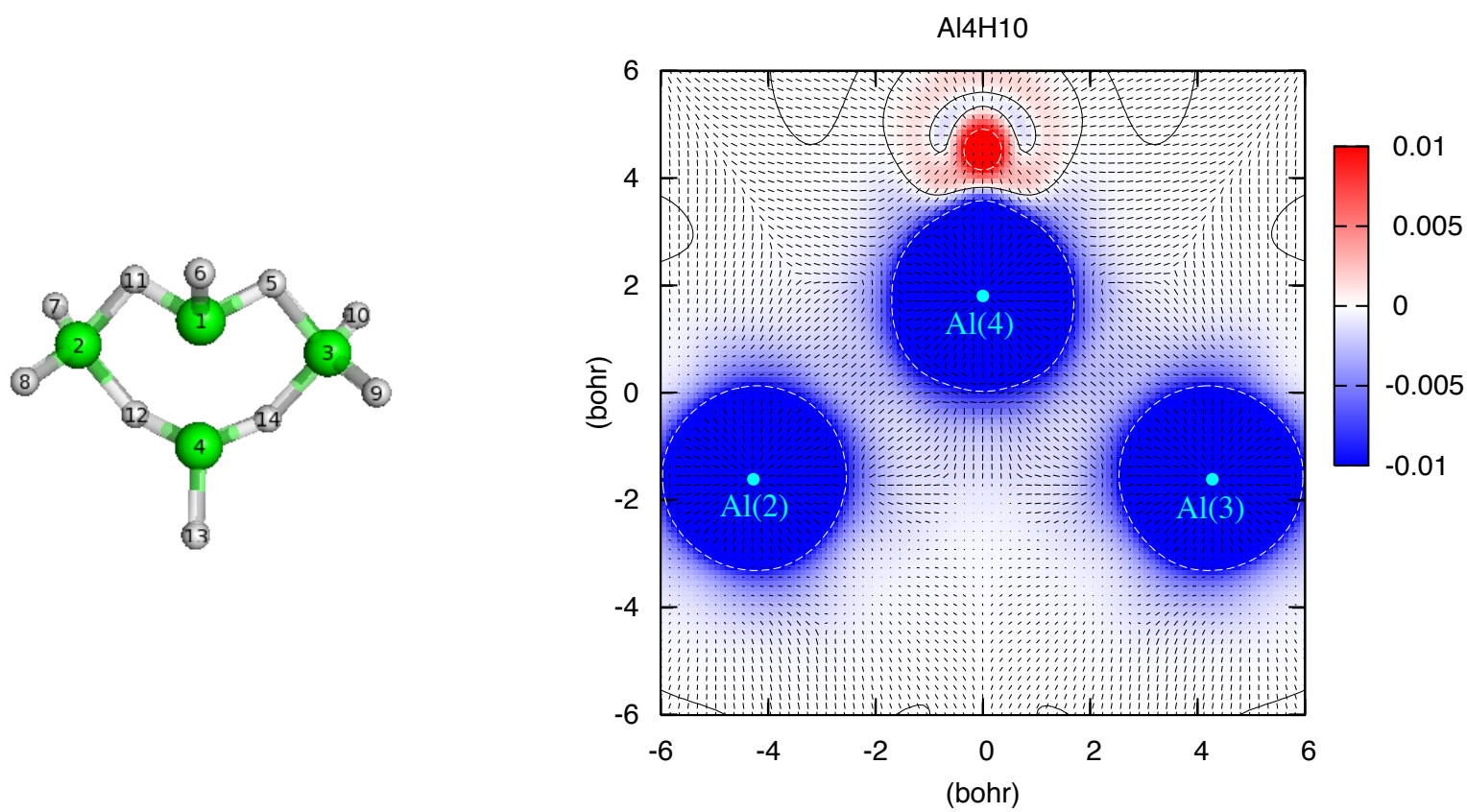

Figure 7: The largest eigenvalue of the stress tensor and corresponding eigenvector of $\mathrm{Al}_{4} \mathrm{H}_{10}$ (but on the different plane from Fig. 5), plotted in the same manner as Fig. 4. 